\title{
Fertilidade do solo e estoques de carbono e nitrogênio sob sistemas agroflorestais no Cerrado Mineiro
}

\author{
Soil fertility and carbon and nitrogen stocks under agroforestry systems in the \\ Cerrado of Minas Gerais State
}

\author{
Juliana Martins Ribeiro ${ }^{I}$, Leidivan Almeida FrazãoII, \\ Paulo Henrique Silveira Cardoso ${ }^{\mathrm{III}}$, Agda Loureiro Gonçalves Oliveira ${ }^{\mathrm{IV}}$, \\ Regynaldo Arruda Sampaio ${ }^{\text {II, }}$ Luiz Arnaldo Fernandes ${ }^{\text {II }}$
}

\begin{abstract}
Resumo
Os sistemas agroflorestais são utilizados para diversificar a produção de alimentos, proporcionar aumento na biodiversidade da fauna e da flora e conservação do carbono e nutrientes no solo. Sendo assim, o objetivo deste estudo foi avaliar a fertilidade e os estoques de carbono (C) e nitrogênio (N) do solo após dez anos de implantação de sistemas agroflorestais (SAFs) no Cerrado mineiro. O estudo foi realizado no Assentamento Agroextrativista Americana, localizado no município de Grão Mogol, mesorregião norte do Estado de Minas Gerais. Foram selecionados três SAFs e duas áreas de vegetação nativa (VN) adjacentes, utilizadas como referência para o estudo, as quais foram agrupados em função da classe de solo: Latossolo Vermelho Amarelo e Cambissolo Háplico. A amostragem do solo foi realizada em março de 2013, dez anos após a implantação dos SAFs, em que amostras compostas foram retiradas das camadas 0-5 e 0-20 cm de profundidade para avaliação dos teores de nutrientes e densidade aparente, e na primeira camada os estoques de $\mathrm{C}$ e $\mathrm{N}$ do solo. Os SAFs que foram implantados com manejo baseado em desrama e manutenção da diversidade florística contribuíram com e melhoria da qualidade do solo e manutenção da fertilidade e estoques de $\mathrm{C}$ em níveis semelhantes à vegetação nativa. Já o SAF que foi implantado com a prévia supressão da vegetação nativa reduziu a diversidade arbórea e, consequentemente, os estoques de C ao longo do tempo. Porém, o manejo com culturas anuais e uso de leguminosas contribuiu para a melhoria da fertilidade do solo e incremento dos teores e estoques de N.
\end{abstract}

Palavras-chave: Diversidade florística; Qualidade do solo; Teores de nutrientes; Relação C:N

\begin{abstract}
Agroforestry systems are used to diversify food production, provide an increase in the biodiversity of fauna and flora as well as the conservation of carbon and nutrients in the soil. Thus, the objective of this study was to evaluate the fertility and carbon $(\mathrm{C})$ and nitrogen $(\mathrm{N})$ stocks of the soil after ten years of agroforestry system (SAFs) implantation in the Cerrado biome of Minas Gerais state, Brazil. The study was carried out in the American Agroextractivist Settlement, located in the municipality of Grão Mogol, northern mesoregion of the state of Minas Gerais. Three SAFs and two adjacent native vegetation areas (VNs) were used as reference for the study, which were grouped according to the soil class: Red-Yellow Latosol and Haplic Cambisol. The soil sampling was carried out in March 2013, ten years after the implementation of the SAFs, where composite samples were removed from the $0-5$ and $0-20 \mathrm{~cm}$ soil depth layers for the evaluation of nutrient contents and apparent density, and in the first layer the soil $\mathrm{C}$ and $\mathrm{N}$ stocks. SAFs that were deployed with management based on prune trees and maintenance of floristic diversity contributed with the improvement of the soil quality and the maintenance of fertility and C stocks at levels like in native vegetation. On the other hand, the SAF that was implanted with the previous suppression of the native vegetation reduced the tree diversity and, consequently, the $C$ stocks over time. However, management with annual crops and the use of legumes contributed to the improvement of soil fertility and to the increase of $\mathrm{N}$ contents and stocks.
\end{abstract}

Keywords: Floristic diversity; Soil quality; Nutrient contents; C:N ratio

Engenheira Agrônoma, MSc., Doutoranda do Instituto de Ciências Agrárias, Universidade Federal de Minas Gerais, Av. Universitária, 1000, CEP 39404-547, Montes Claros (MG), Brasil. juliannaribeiromartins@ hotmail.com (ORCID: 0000-0001-6131-4992)

II Engenheiro(a) Agrônomo(a), Dr.(a), Professor(a) do Instituto de Ciências Agrárias, Universidade Federal de Minas Gerais, Av. Universitária, 1000, CEP 39404-547, Montes Claros (MG), Brasil. lafrazao@ica.ufmg.br (ORCID: 0000-0001-6848-9007) / larnaldo@ufmg.br (ORCID: 0000-0002-98771924) / rsampaio@ica.ufmg.br (ORCID: 0000-0003-3214-6111)

III Engenheiro Agrícola e Ambiental, MSc., Doutorando do Centro de Energia Nuclear na Agricultura, Universidade de São Paulo, Av. Centenário, 303 CEP 13400-970, Piracicaba (SP), Brasil. paulohenrique.sc@ hotmail.com (ORCID: 0000-0002-6209-7026)

IV Engenheira Agrícola e Ambiental, Mestranda em Engenharia Agrícola da Universidade Estadual de Campinas, Av. Candido Rondon, 501,CEP 13083-875, Campinas (SP), Brasil. agdaloureiro@gmail.com (ORCID: 0000-0002-8761-9716) 
Ribeiro, J. M.; Frazão, L. A.; Cardoso, P. H. S.; Oliveira, A. L. G.; Sampaio, R. A.;

\section{Introdução}

O desempenho dos sistemas agroflorestais (SAFs) pode ser relacionado com a quantidade de nutrientes liberados durante o processo de decomposição do material orgânico aportado ao solo (SILVA, 2011) e com a capacidade de reter carbono no solo, minimizando a sua perda para a atmosfera (SILVA; ARAÚJO; KUSDRA, 2014). Esses sistemas apresentam inúmeras vantagens que contribuem para o estabelecimento de modelos de produção agrícola mais sustentável, com redução dos problemas relacionados ao manejo e à conservação do solo, principalmente em regiões de transição para o semiárido, que apresentam um longo período de estiagem.

Comparativamente aos sistemas convencionais de cultivo, os SAFs proporcionam maior cobertura do solo, favorecem a preservação da fauna e da flora, promovem a ciclagem de nutrientes a partir da ação de sistemas radiculares diversos e propiciam um contínuo aporte de matéria orgânica (MAIA et al., 2006), além de uma maior diversidade de produtos a serem explorados comercialmente.

Em regiões tropicais, os SAFs podem ser eficientes sequestradores de carbono (C) tanto pela alta produtividade de biomassa quanto pelo significativo aumento dos estoques de carbono no solo (FROUFE; RACHWAL; SEOANE, 2011). O C orgânico do solo é um importante indicador de qualidade e de sustentabilidade dos sistemas nativos (IWATA, 2010), e a sua determinação conjuntamente com os teores de nitrogênio $(\mathrm{N})$ e de nutrientes do solo pode auxiliar no direcionamento de estratégias de manejo para os agroecossistemas.

As mudanças no conteúdo de $\mathrm{C}$ do solo resultam também em alterações nos conteúdos de N. Esses dois elementos são fonte de energia para a biomassa microbiana, que participa no armazenamento e ciclagem de nutrientes e na melhoria das propriedades físicas e químicas dos solos (IWATA, 2010). A baixa disponibilidade de $\mathrm{N}$ no solo, associada à grande demanda pelas plantas, pode limitar o crescimento e desenvolvimento das espécies nativas e cultivadas (SANTIAGO et al., 2013). Sendo assim, a entrada de C e N pela adição de matéria orgânica é essencial para a manutenção da biomassa microbiana do solo, uma vez que os micro-organismos são os principais responsáveis pela ciclagem desses elementos no solo (PITOMBO, 2011).

Diante do exposto, o objetivo deste trabalho foi quantificar os teores de nutrientes e os estoques de C e N na camada superficial do solo sob Sistemas Agroflorestais (SAFs) e respectivas vegetações nativas adjacentes, em áreas de agricultores familiares localizadas na mesorregião norte do Estado de Minas Gerais.

\section{Material e métodos}

\section{Caracterização da área de estudo}

O estudo foi realizado no Assentamento Agroextrativista Americana, localizado no município de Grão Mogol, mesorregião norte do estado de Minas Gerais, com coordenada

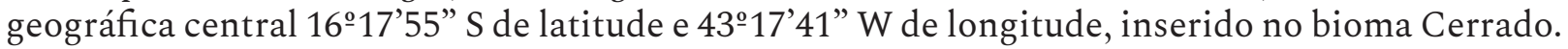
O clima da região, de acordo com a classificação de Köppen, é tropical seco, com precipitação pluviométrica média anual de 1000 a $1300 \mathrm{~mm}$, inverno seco e temperatura média anual superior a $18^{\circ} \mathrm{C}$ (ALVARES et al., 2013).

O assentamento abriga 75 famílias em uma área de aproximadamente 18 mil hectares. Para este estudo foram selecionados três Sistemas Agroflorestais (SAFs) de um hectare, os quais foram divididos em dois grupos em função da classe de solo: Latossolo Vermelho Amarelo e Gleissolo Háplico.

Os SAFs 1 e 2 foram implantados em 2003 em uma área de Cerrado denso sob Latossolo Vermelho Amarelo, localizado na meia encosta com relevo suave ondulado. Na implantação desses sistemas, foram preservadas as espécies vegetais nativas estratégicas, como melíferas, 
madeireiras, frutíferas e medicinais. As espécies arbóreas preservadas foram desbastadas e/ou desramadas para possibilitar a entrada de luz e a introdução de espécies de interesse. O critério de seleção das espécies introduzidas foi em função da fertilidade natural do solo, tolerância ao estresse hídrico e aceitação no mercado local.

O SAF3 também foi implantado em 2003 em área Mata de Galeria sob Gleissolo Háplico, na mesma encosta dos SAFs 1 e 2, porém, em relevo plano no qual, antigamente, era constituída uma várzea. No início da década de 1980, essa área foi drenada e a sua vegetação nativa suprimida para cultivo. Durante os anos de cultivo não se fizeram usos de insumos químicos. Essa atividade ocorreu até o início da década de 1990, quando a área foi abandonada. Em 2003, quando foi introduzido o SAF3, as espécies nativas predominantes eram: o araçazeiro (Psidium firmum $\mathrm{O}$ Berg.) e a goiabeira (Psidium guajava), que foram mantidas em função do interesse comercial de seus frutos. As duas espécies são secundárias e tem grande capacidade de regeneração na área de estudo. Nesse sistema, a escolha das espécies introduzidas foi em função da tolerância à luminosidade e do tipo de solo. O manejo das espécies arbóreas foi realizado por meio de desramas para aumentar a disponibilidade de luz no sistema e adicionar matéria orgânica ao solo (ROCHA et al., 2014).

Para fins de comparação foram avaliadas duas áreas de vegetação nativa de Cerrado (VN) adjacentes, utilizadas como referência da condição original do solo (testemunha), denominadas de VN1 (para os SAFs 1 e 2) e VN2 (para o SAF3).

De acordo com o levantamento florístico realizado por Rocha et al. (2014) foi possível constatar uma variabilidade no número famílias, espécies e indivíduos arbóreos lenhosos por hectare em cada SAF e VN avaliadas (Tabela 1). Os SAFs 1 e 2 possuíam maior diversidade florística do que o SAF3, assim como a VN1 apresenta maior número de famílias, espécies e indivíduos em relação a VN2.

\section{Tabela 1 - Família e número de espécies (ESP) e indivíduos arbóreos lenhosos (IND) em sistemas agroflorestais (SAF) e vegetação nativa adjacentes (VN) localizados em Grão Mogol-MG. Adaptado de Rocha et al. (2014).}

Table 1 - Family and number of species (ESP) and woody individuals (IND) in agroforestry systems (SAF) and adjacent native vegetation (VN) located in Grão Mogol/MG state. Adapted from Rocha et al. (2014).

\begin{tabular}{lcccccccccc}
\hline \multicolumn{1}{c}{ Família } & \multicolumn{2}{c}{ SAF1 } & \multicolumn{2}{c}{ SAF2 } & \multicolumn{2}{c}{ VN1 } & \multicolumn{2}{c}{ SAF3 } & \multicolumn{2}{c}{ VN2 } \\
& ESP & IND & ESP & IND & ESP & IND & ESP & IND & ESP & IND \\
\hline Anacardiaceae & 02 & 180 & 02 & 120 & 02 & 100 & - & - & 02 & 440 \\
Annonaceae & 02 & 40 & - & - & - & - & - & - & - & - \\
Bignoniaceae & - & - & 02 & 80 & 02 & 120 & - & - & 01 & 40 \\
Bixaceae & 01 & 40 & - & - & - & - & - & - & - & - \\
Bombacaceae & - & - & - & - & 01 & 60 & - & - & - & - \\
Caricaceae & 01 & 20 & - & - & - & - & - & - & - & - \\
Combretaceae & 01 & 40 & 01 & 20 & 01 & 40 & - & - & - & - \\
Euphorbiaceae & 01 & 20 & - & - & - & - & - & - & - & - \\
Fabaceae & 06 & 240 & 02 & 40 & 01 & 100 & - & - & 01 & 20 \\
Lamiaceae & - & - & 01 & 60 & - & - & - & - & - & - \\
& & & & & & & & & Continua...
\end{tabular}


Tabela 1 - Conclusão...

Table 1 - Conclusion...

\begin{tabular}{|c|c|c|c|c|c|c|c|c|c|c|}
\hline \multirow[t]{2}{*}{ Família } & \multicolumn{2}{|c|}{ SAF1 } & \multicolumn{2}{|c|}{ SAF2 } & \multicolumn{2}{|c|}{ VN1 } & \multicolumn{2}{|c|}{ SAF3 } & \multicolumn{2}{|c|}{ VN2 } \\
\hline & ESP & IND & ESP & IND & ESP & IND & ESP & IND & ESP & IND \\
\hline Loganiaceae & 01 & 20 & - & - & - & - & - & - & - & - \\
\hline Malpighiaceae & 01 & 20 & - & - & - & - & - & - & 01 & 60 \\
\hline Moraceae & 01 & 20 & 01 & 60 & - & - & - & - & - & - \\
\hline Musaceae & 01 & 80 & - & - & - & - & - & - & - & - \\
\hline Myrtaceae & 01 & 40 & 01 & 40 & 01 & 40 & 02 & 440 & 02 & 280 \\
\hline Nyctaginaceae & - & - & - & - & 01 & 80 & - & - & - & - \\
\hline Palmaceae & 01 & 40 & - & - & 01 & 360 & - & - & - & - \\
\hline Rubiaceae & - & - & - & - & 01 & 20 & - & - & - & - \\
\hline Rutaceae & 02 & 40 & - & - & 01 & 20 & - & - & 01 & 20 \\
\hline Sapindaceae & 01 & 120 & 01 & 60 & 01 & 140 & - & - & - & - \\
\hline Tiliaceae & 01 & 20 & 01 & 20 & - & - & - & - & - & - \\
\hline Vochysiaceae & 01 & 20 & 01 & 60 & 01 & 40 & - & - & - & - \\
\hline Total de ESP e IND & 25 & 1000 & 13 & 560 & 14 & 1120 & 02 & 440 & 08 & 860 \\
\hline
\end{tabular}

Dentre as espécies de valor comercial encontradas e/ou cultivadas nos SAFs, pode-se dar destaque para Ananascomosus, Malpighiae marginata, Psidium cattleyanum, Musa spp., Psidium guajava, Annona muricata, Citruslimon, Caricapapaya, Mangifera indica, Byrsonima crassifólia, Annona crassiflora e Bixa orellana. Especificamente no SAF3, em que há maior incidência de radiação solar e baixa diversidade florística, há o cultivo de culturas anuais como feijão (Phaseolus vulgaris) e milho (Zea mays) em sistemas consorciados e/ ou rotacionados.

\section{Amostragem do solo}

A amostragem do solo foi realizada 10 anos após a implantação dos SAFs, no mês de março de 2013, época caracterizada pela estação chuvosa, com temperaturas médias acima de $22{ }^{\circ} \mathrm{C}$. As amostras foram coletadas na camada $0-5 \mathrm{~cm}$ de profundidade, com uma pá de corte, para realizar as análises químicas e granulométricas. Nessa camada, há maior influência do material orgânico depositado na superfície do solo (serapilheira). Na profundidade de $0-20 \mathrm{~cm}$ do solo foram coletadas amostras com trado holandês para determinação dos atributos químicos e avaliação da fertilidade do solo. Em cada sistema e profundidade avaliados foram coletadas três amostras compostas de solo, em que cada uma delas foi originada de cinco amostras simples coletadas aleatoriamente nos locais de estudo.

A densidade aparente do solo foi determinada pelo método do anel volumétrico, utilizandose um cilindro de $5 \mathrm{~cm}$ de diâmetro por $5 \mathrm{~cm}$ de altura, e coletando-se amostras indeformadas nas camadas de $0-5,5-10,10-15$ e $15-20 \mathrm{~cm}$ de profundidade. O valor de densidade aparente para a profundidade de $0-20 \mathrm{~cm}$ foi obtido pela média das densidades nas camadas amostradas.

Análises químicas, físicas e teores de carbono $(\mathrm{C})$ e nitrogênio $(\mathrm{N})$

As análises químicas para a avaliação da fertilidade, determinação das frações granulométricas e da densidade aparente do solo foram realizadas em duplicatas, conforme Embrapa (1997), enquanto que os teores de nitrogênio mineral ( $\mathrm{N}$ mineral) foram determinados 
de acordo com metodologia proposta por Alves et al. (1994).

A determinação dos teores de $\mathrm{C}$ orgânico total e de $\mathrm{N}$ total foi realizada em duplicatas, somente na camada de $0-5 \mathrm{~cm}$ de profundidade. As amostras de solo foram previamente secas ao ar, homogeneizadas, moídas e passadas em peneiras de $0,150 \mathrm{~mm}$ e, posteriormente, analisadas por combustão seca no equipamento LECO CN-2000. Com os valores obtidos para os teores de $\mathrm{C}$ e $\mathrm{N}$ calculou-se a relação $\mathrm{C}: \mathrm{N}$ do solo.

Os estoques de $\mathrm{C}$ e $\mathrm{N}$ foram calculados pela multiplicação dos teores de cada elemento (C ou N) pela densidade aparente do solo e camada amostrada $(0-5 \mathrm{~cm}$ de profundidade).

Os valores de cada variável foram submetidos ao teste de Lilliefors para verificar se os dados das análises apresentavam distribuições normais. Aplicou-se também o teste de Cochran e Bartlett para verificar a homogeneidade de variâncias. Como os tratamentos avaliados encontravam-se dispostos lateralmente (sem aleatorização) e as hipóteses de normalidade e homogeneidade não foram validadas, optou-se pelo uso de estatística não paramétrica para todas as variáveis. Assim, foi aplicado o teste Kruskal-Wallis $(\mathrm{p}<0,05)$, utilizando-se o software $\mathrm{R}$ (R Development Core Team).

\section{Resultados e discussão}

De acordo com o triângulo textural de Atterberg, os solos dos Sistemas Agroflorestais 1 e 2 (SAF1 e SAF2) e de vegetação nativa adjacente (VN1), são enquadrados na classe textural francoargilo-arenosa, enquanto os solos do SAF3 e da VN2 na classe textural argilo-arenosa (Tabela 2). Os teores de argila do Gleissolo Háplico, em que estão o SAF3 e a VN2, foram superiores àqueles encontrados no Latossolo Vermelho Amarelo, onde estão o SAF1, SAF2 e a VN1.

Tabela 2 - Composição granulométrica e densidade do solo sob sistemas agroflorestais (SAFs) e vegetação nativa adjacentes (VNs) em Grão Mogol-MG.

Table 2 - Soil granulometric composition and bulk density under agroforestry systems (SAFs) and adjacent native vegetation (VNs) in the municipality of Grão Mogol/MG state.

\begin{tabular}{|c|c|c|c|c|c|c|c|c|}
\hline \multirow{3}{*}{ Sistemas } & \multicolumn{2}{|c|}{ Areia } & \multicolumn{2}{|c|}{ Silte } & \multicolumn{2}{|c|}{ Argila } & \multirow{2}{*}{\multicolumn{2}{|c|}{$\begin{array}{c}\begin{array}{c}\text { Densidade } \\
\text { Aparente }\end{array} \\
\mathrm{kg} \mathrm{dm}^{-3}\end{array}$}} \\
\hline & \multicolumn{6}{|c|}{$\mathrm{g} \mathrm{kg}^{-1}$} & & \\
\hline & $0-5 \mathrm{~cm}$ & $0-20 \mathrm{~cm}$ & $0-5 \mathrm{~cm}$ & $0-20 \mathrm{~cm}$ & $0-5 \mathrm{~cm}$ & $0-20 \mathrm{~cm}$ & $0-5 \mathrm{~cm}$ & $0-20 \mathrm{~cm}$ \\
\hline SAF1 & $530 a^{*}$ & $520 \mathrm{a}$ & $140 \mathrm{~b}$ & $140 \mathrm{~b}$ & $330 \mathrm{~b}$ & $340 \mathrm{~b}$ & $1,13 \mathrm{a}$ & $1,15 \mathrm{a}$ \\
\hline SAF2 & $470 \mathrm{a}$ & $440 \mathrm{ab}$ & $170 \mathrm{~b}$ & $180 \mathrm{~b}$ & $350 \mathrm{~b}$ & $350 \mathrm{~b}$ & $1,17 \mathrm{a}$ & $1,16 \mathrm{a}$ \\
\hline VN1 & $540 \mathrm{a}$ & $540 \mathrm{a}$ & $150 \mathrm{~b}$ & $160 \mathrm{~b}$ & $310 \mathrm{~b}$ & $300 \mathrm{~b}$ & $1,14 \mathrm{a}$ & $1,15 \mathrm{a}$ \\
\hline SAF3 & $410 \mathrm{~b}$ & $420 \mathrm{~b}$ & $230 \mathrm{a}$ & $220 \mathrm{a}$ & $370 \mathrm{a}$ & $370 \mathrm{a}$ & $1,15 \mathrm{a}$ & $1,16 \mathrm{a}$ \\
\hline VN2 & $400 \mathrm{~b}$ & $380 \mathrm{~b}$ & $230 \mathrm{a}$ & $220 \mathrm{a}$ & $370 \mathrm{a}$ & $400 \mathrm{a}$ & $1,14 \mathrm{a}$ & $1,14 \mathrm{a}$ \\
\hline
\end{tabular}

* Médias seguidas pelas mesmas letras, nas linhas, para cada atributo e profundidade avaliada, não diferem entre si pelo teste de Kruskal-Wallis $(\mathrm{p}<0,05)$.

A densidade aparente do solo foi semelhante entre os sistemas avaliados para os dois tipos de solo e camadas amostradas (Tabela 2). Esse atributo físico do solo é fortemente afetado pelo manejo e pela matéria orgânica do solo adicionada (LEAL FILHO et al., 2013). Sendo assim, pode-se observar que a condução dos SAFs com aporte de material vegetal por dez anos consecutivos contribuiu para o processo de agregação e manutenção da porosidade do solo, de forma que a densidade ficou em níveis semelhantes aos observados nas duas áreas de vegetação 
nativa avaliadas.

A matéria orgânica depositada na superfície do solo pode alterar o arranjo das partículas primárias do solo e, consequentemente, a densidade do solo. Alvarenga et al. (2015), estudando os mesmos sistemas, verificaram que, ao longo do ano, as quantidades serapilheira depositada na superfície do Latossolo Vermelho Amarelo foram maiores nos SAF1 (18.191 kg ha-1 ano-1) e SAF2 (24.904 kg ha-1 ano ${ }^{-1}$ em relação a VN1 (15.803 kg ha-1 ano-1), enquanto que no Gleissolo Háplico, a VN2 (19.269 kg ha-1 ano $\left.^{-1}\right)$ depositou maiores quantidades em relação ao SAF3 $\left(15.037 \mathrm{~kg} \mathrm{ha}^{-1} \mathrm{ano}^{-1}\right)$. Segundo os autores, as diferenças nas quantidades de material depositado estão relacionadas à composição florística das áreas e ao manejo dos SAFs por meio de desramas.

Embora os SAFs e VNs tenham apresentado diferenças quanto ao número de famílias, número de espécies e indivíduos arbóreos, estes dados não influenciaram a densidade do solo após 10 anos de implantação dos sistemas agroflorestais (Tabela 2). Apesar de haver um menor número de indivíduos e espécies arbóreas no SAF3 e menor deposição de material orgânico (ROCHA et al., 2014) ao longo dos anos, o acúmulo de matéria orgânica, associada ao manejo do solo, tem contribuído para a manutenção dos valores de densidade do solo a VN3.

Matias et al. (2009) verificaram menores valores de densidade do solo em mata nativa do que em áreas ocupadas com monocultivos de milho e pastagem. Os autores atribuíram os achados à maior quantidade de matéria orgânica, teor de argila e ausência de tráfego de máquinas agrícolas, o que também foi verificado neste estudo.

Tabela 3 - Teores de nutrientes do solo sob sistemas agroflorestais (SAFs) e vegetação nativa adjacentes (VNs) em Grão Mogol-MG.

Table 3 - Soil nutrient contents under agroforestry systems (SAFs) and adjacent native vegetation (VNs) in the municipality of Grão Mogol/MG state.

\begin{tabular}{|c|c|c|c|c|c|c|c|c|c|c|}
\hline \multirow{3}{*}{ Sistemas } & \multirow{2}{*}{\multicolumn{2}{|c|}{ 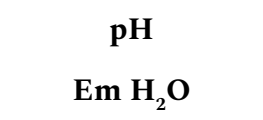 }} & \multicolumn{2}{|c|}{ Cálcio } & \multicolumn{2}{|c|}{ Magnésio } & \multicolumn{2}{|c|}{ Potássio } & \multicolumn{2}{|c|}{ Alumínio } \\
\hline & & & \multicolumn{8}{|c|}{$\mathrm{mmol}_{c} \mathrm{dm}^{-3}$} \\
\hline & $0-5 \mathrm{~cm}$ & $0-20 \mathrm{~cm}$ & $0-5 \mathrm{~cm}$ & $0-20 \mathrm{~cm}$ & $0-5 \mathrm{~cm}$ & $0-20 \mathrm{~cm}$ & $0-5 \mathrm{~cm}$ & $0-20 \mathrm{~cm}$ & $0-5 \mathrm{~cm}$ & $0-20 \mathrm{~cm}$ \\
\hline SAF1 & $5,3 b^{*}$ & $5,8 \mathrm{a}$ & $63 a$ & $38 \mathrm{~b}$ & $24 \mathrm{a}$ & $17 \mathrm{~b}$ & $3,5 \mathrm{a}$ & $3,3 \mathrm{a}$ & $0 \mathrm{a}$ & $0 \mathrm{a}$ \\
\hline SAF2 & $5,3 \mathrm{~b}$ & $5,7 \mathrm{a}$ & $58 \mathrm{a}$ & $33 \mathrm{~b}$ & $25 \mathrm{a}$ & $16 \mathrm{~b}$ & $4,2 \mathrm{a}$ & $3,2 \mathrm{~b}$ & $0 \mathrm{a}$ & $0 \mathrm{a}$ \\
\hline VN1 & $5,1 \mathrm{~b}$ & $5,8 \mathrm{a}$ & $56 a$ & $46 \mathrm{~b}$ & $35 \mathrm{a}$ & $21 \mathrm{~b}$ & $5,8 \mathrm{a}$ & $3,2 \mathrm{~b}$ & $0 \mathrm{a}$ & $0 \mathrm{a}$ \\
\hline SAF3 & $5,3 \mathrm{~b}$ & $5,8 \mathrm{a}$ & $41 \mathrm{a}$ & $18 \mathrm{~b}$ & $31 \mathrm{a}$ & $11 \mathrm{~b}$ & $4,3 \mathrm{a}$ & $1,8 \mathrm{~b}$ & $1,0 \mathrm{a}$ & $1,0 \mathrm{a}$ \\
\hline \multirow[t]{2}{*}{ VN2 } & $5,2 \mathrm{~b}$ & $5,6 \mathrm{a}$ & $41 \mathrm{a}$ & $23 \mathrm{~b}$ & $32 \mathrm{a}$ & $15 \mathrm{~b}$ & $12,8 \mathrm{a}$ & $2,2 \mathrm{~b}$ & $4,0 \mathrm{a}$ & $2,0 \mathrm{~b}$ \\
\hline & \multicolumn{2}{|c|}{ Fósforo } & \multicolumn{2}{|c|}{ Enxofre } & \multicolumn{2}{|c|}{$\begin{array}{c}\text { Boro } \\
\mathbf{m m o l}_{c} \mathbf{d m}^{-3}\end{array}$} & \multicolumn{2}{|c|}{ Zinco } & \multicolumn{2}{|c|}{$\mathbf{N}$ mineral } \\
\hline & $0-5 \mathrm{~cm}$ & $0-20 \mathrm{~cm}$ & $0-5 \mathrm{~cm}$ & $0-20 \mathrm{~cm}$ & $0-5 \mathrm{~cm}$ & $0-20 \mathrm{~cm}$ & $0-5 \mathrm{~cm}$ & $0-20 \mathrm{~cm}$ & $0-5 \mathrm{~cm}$ & $0-20 \mathrm{~cm}$ \\
\hline SAF1 & $2,4 \mathrm{a}$ & $0,8 \mathrm{~b}$ & $8,3 \mathrm{a}$ & $3,4 \mathrm{~b}$ & $0,5^{\mathrm{ns}}$ & 0,4 & $1,2 \mathrm{a}$ & $1,3 \mathrm{a}$ & $47 \mathrm{a}$ & $12 \mathrm{~b}$ \\
\hline SAF2 & $1,7 \mathrm{a}$ & $0,5 \mathrm{~b}$ & $12,5 \mathrm{a}$ & $5,7 \mathrm{~b}$ & 0,6 & 0,5 & $1,5 \mathrm{a}$ & $1,4 \mathrm{a}$ & $45 \mathrm{a}$ & $14 \mathrm{~b}$ \\
\hline VN1 & $1,5 \mathrm{a}$ & $0,3 \mathrm{~b}$ & $12,2 \mathrm{a}$ & $3,3 \mathrm{~b}$ & 0,6 & 0,8 & $1,6 \mathrm{a}$ & $1,6 \mathrm{a}$ & $62 \mathrm{a}$ & $14 \mathrm{~b}$ \\
\hline SAF3 & $2,1 \mathrm{a}$ & $0,4 \mathrm{~b}$ & $10,7 \mathrm{a}$ & $4,5 \mathrm{~b}$ & 0,8 & 1,0 & $1,0 \mathrm{a}$ & $0,9 \mathrm{a}$ & $69 a$ & $23 \mathrm{~b}$ \\
\hline VN2 & $2,8 \mathrm{a}$ & $1,1 \mathrm{~b}$ & $13,4 \mathrm{a}$ & $2,4 \mathrm{~b}$ & 0,3 & 0,5 & $0,9 \mathrm{a}$ & $0,4 \mathrm{~b}$ & $61 \mathrm{a}$ & $10 \mathrm{~b}$ \\
\hline
\end{tabular}

* Médias seguidas pelas mesmas letras em cada linha, entre as colunas para cada atributo avaliado, não diferem estatisticamente entre as profundidades avaliadas pelo teste de Kruskal-Wallis ( $\mathrm{p}<0,05)$. ${ }^{\text {ns }}$ Não significativo para o atributo avaliado. 
A fertilidade dos solos dos SAFs e VNs, de modo geral, foi semelhante para os atributos avaliados, sendo que os teores de nutrientes foram superiores na camada $0-5 \mathrm{~cm}$ de profundidade (Tabela 3). A mineralização do material orgânico depositado na superfície dos solos por 10 anos contribuiu para o aumento dos teores de nutrientes na camada superficial do solo.

A acidez ativa dos solos foi classificada como acidez média e os valores de $\mathrm{pH}$ foram superiores na camada de 0-20 cm. De acordo com classificação proposta por Alvarez et al. (1999), os teores de $\mathrm{Ca}, \mathrm{Mg}$ e $\mathrm{K}$ na camada $0-5 \mathrm{~cm}$ de profundidade foram classificados como muito bom, enquanto que, na camada $0-20 \mathrm{~cm}$, variaram de médio a bom. Já os teores de $\mathrm{P}$ e Al trocável, em todos os sistemas e camadas avaliadas, foram classificados como muito baixo.

Os teores de $S$ foram classificados como bom, na camada $0-5 \mathrm{~cm}$ e baixo a médio, na camada 0-20 cm de profundidade (ALVAREZ et al., 1999). Os teores de $\mathrm{N}$ mineral (nitrato e amônio) também foram superiores na camada $0-5 \mathrm{~cm}$, demonstrando que há maior ciclagem de nutrientes na camada superficial, principalmente devido ao aporte de constante de material vegetal. Esses resultados são semelhantes àqueles encontrados por Silva e Vale (2000), em solos classificados como Latossolos e Gleissolos. Os micronutrientes boro e zinco, independentemente da camada de solo amostrada, variaram de baixo a médio, segundo a classificação de Alvarez et al. (1999).

A presença do componente arbóreo nos SAFs favorece uma maior deposição de material vegetal ao solo (raízes, galhos, folhas), contribuindo para a melhoria da fertilidade do solo pelo processo de mineralização dos nutrientes (CAMPANHA; SANTOS, 2007). Neste estudo verificaram-se maiores teores de nutrientes na camada superficial do solo (Tabela 3), principalmente pelo aporte constante de material orgânico proveniente da desrama (SAFs), do estrato arbóreo e arbustivo (VN) e pela renovação do sistema radicular das plantas nativas e cultivadas.

Os teores e estoques de $\mathrm{C}$ do solo na camada $0-5 \mathrm{~cm}$ de profundidade foram maiores nos SAFs implantados sob o Latossolo Vermelho Amarelo (Tabela 4). Os teores e estoques de C semelhantes a VN1 podem ser explicados pela diversidade florística do local, que aumenta a qualidade do material orgânico adicionado, e consequentemente, os processos de humificação que contribuem para o armazenamento de $\mathrm{C}$ ao longo prazo. Conforme pode ser constatado no estudo de Rocha et al. (2014) nas mesmas áreas deste estudo, as diferenças na qualidade do material depositado ao solo estão relacionadas à composição florística das áreas e ao manejo adotado aos SAFs por meio de podas.

Os resultados evidenciaram que a diversidade vegetal e o manejo utilizado no SAF1 e SAF2 têm contribuído para a manutenção e estabilidade do carbono no solo, uma vez que ocorre o aporte de resíduos ao longo dos anos e não ocorrem revolvimento e ações perturbadoras ao solo. Esses achados demonstram o potencial dos sistemas agroflorestais para restabelecimento do equilíbrio e melhoria da ciclagem de nutrientes ao longo do tempo, contribuindo para o aumento dos teores de matéria orgânica do solo e aportes de C e N (IWATA et al., 2012). De acordo com Bochner et al. (2008) e Silva et al. (2012), os teores elevados de C em áreas florestadas se devem ao maior tempo de cobertura florestal e ao maior aporte de serapilheira. Assim como observado no presente estudo, Gama-Rodrigues et al. (2010) verificaram que o estoque de C do solo não diferiu entre os SAFs estudados e a floresta nativa, evidenciando, segundo esses autores, a importância dos sistemas agroflorestais para a manutenção e incremento do C no solo.

Nas áreas sob Gleissolo Háplico, o SAF3 apresentou menores teores e estoques de C que a VN2 (Tabela 4). Esses resultados podem ser explicados pelo histórico da área do SAF3, que teve a vegetação nativa totalmente suprimida para o cultivo na década de 1980. Após abandonada, na década de 1990, as únicas duas espécies arbóreas que regeneraram, de acordo com Rocha et al. (2014), foram o araçazeiro (Psidium firmum O Berg.) e a goiabeira (Psidium guajava).O menor número de diversidade de espécies arbóreas, bem como a menor densidade de plantas para favorecer a entrada de luz no sistema com o objetivo de cultivar espécies anuais mais exigentes em luminosidade, como milho e feijão, certamente estão contribuindo para a menor estocagem de $\mathrm{C}$ ao longo do tempo. 
Os menores estoques de C no SAF3 podem ser também atribuídos ao aumento no consumo do C prontamente disponível pela biomassa microbiana do solo e por meio da exportação de C pelas culturas anuais. Alvarenga et al. (2015), trabalhando nessa mesma área e com os mesmos sistemas, verificaram que a composição da serapilheira do SAF3 era mais rica em lignina e celulose que a da VN2, dificultando a decomposição do material depositado pela comunidade microbiana e, consequentemente, o aporte de C. Resultados semelhantes ao deste estudo foram encontrados por Iwata et al. (2012), onde SAFs com seis anos de implantação apresentaram baixos estoques de $\mathrm{C}$ em relação à floresta natural, uma vez que antes da sua implantação teve a vegetação nativa cortada e queimada, com grande liberação de $\mathrm{C}$ na forma de $\mathrm{CO}_{2}$ para a atmosfera e perda de nutrientes.

\section{Tabela 4 - Teores e estoques de carbono e de nitrogênio e relação C: $\mathrm{N}$ do solo $(0-5 \mathrm{~cm}$ de profundidade) em sistemas agroflorestais (SAFs) e respectivas áreas de vegetação nativa adjacentes (VNs) em Grão Mogol-MG.}

Table 4 -Soil carbon and nitrogen contents, stocks, and C: $\mathrm{N}$ ratio $(0-5 \mathrm{~cm}$ soil depth $)$ in agroforestry systems (SAFs) and their respective adjacent native vegetation areas (VNs) in the municipality of Grão Mogol/MG state.

\begin{tabular}{lccccc}
\hline \multirow{2}{*}{ Sistemas } & \multicolumn{2}{c}{ Carbono } & \multicolumn{2}{c}{ Nitrogênio } & \\
\cline { 2 - 5 } & Teor & Estoque & Teor & Estoque & Relação C:N \\
\cline { 2 - 5 } & $\mathbf{g ~ k g}^{-1}$ & $\mathbf{M g ~ h a}^{-1}$ & $\mathbf{g ~ k g}^{-1}$ & $\mathbf{M g ~ h a}^{-1}$ & \\
\hline SAF1 & $62,70 \mathrm{a}^{*}$ & $35,27 \mathrm{a}$ & $4,18 \mathrm{~b}$ & $2,35 \mathrm{~b}$ & $14,99 \mathrm{a}$ \\
SAF2 & $64,73 \mathrm{a}$ & $37,87 \mathrm{a}$ & $4,18 \mathrm{~b}$ & $2,45 \mathrm{~b}$ & $15,47 \mathrm{a}$ \\
VN1 & $66,25 \mathrm{a}$ & $37,76 \mathrm{a}$ & $4,21 \mathrm{~b}$ & $2,40 \mathrm{~b}$ & $15,74 \mathrm{a}$ \\
SAF3 & $60,70 \mathrm{~b}$ & $34,75 \mathrm{~b}$ & $4,93 \mathrm{a}$ & $2,82 \mathrm{a}$ & $12,32 \mathrm{a}$ \\
VN2 & $68,43 \mathrm{a}$ & $39,18 \mathrm{a}$ & $5,41 \mathrm{a}$ & $3,10 \mathrm{a}$ & $12,66 \mathrm{a}$ \\
\hline
\end{tabular}

* Médias seguidas pelas mesmas letras, nas linhas, para o mesmo parâmetro avaliado, não diferem entre si pelo teste de Kruskal-Wallis $(\mathrm{p}<0,05)$.

Quanto aos teores e estoques de $\mathrm{N}$ não houve diferença entre as áreas avaliadas, porém, observou-se uma tendência de aumento dos teores no SAF3 e VN2. Esses resultados podem ser explicados pela composição química do material depositado, decorrente das espécies vegetais encontradas em cada sistema. De acordo com Pulrolnik et al. (2009), substratos orgânicos de decomposição rápida tendem a apresentar teores mais elevados de $\mathrm{N}$ do que aqueles de decomposição mais lenta, responsáveis pela conservação do C no solo. Iwata et al. (2012) observaram teores superiores de $\mathrm{N}$ nos SAFs em detrimento da floresta nativa e inferiores em solos de agricultura convencional em virtude de perdas da matéria orgânica nesse sistema

A relação C:N não se diferenciou entre os SAFs 1 e 2 e a VN1 e entre o SAF3 e a VN2 (Tabela 4). Por outro lado, quando se comparam todas as áreas, no Latossolo Vermelho Amarelo pode-se verificar que os valores foram mais elevados, possivelmente pela composição química do material depositado pelas espécies vegetais presentes. A relação C: $\mathrm{N}$ alta indica uma menor taxa de mineralização da MOS e, consequentemente, conservação do C. Adicionalmente, é importante ressaltar que no SAF3 o cultivo do feijão (Phaseolus vulgaris), planta leguminosa, pode favorecer a simbiose com bactérias fixadoras de nitrogênio atmosférico $\left(\mathrm{N}_{2}\right)$ e produção de resíduos culturais ricos em nitrogênio, contribuindo para diminuição da relação C:N nesta área.

Outros estudos também correlacionaram os aumentos dos teores de C com o aporte de matéria orgânica proveniente da deposição contínua e variada de substratos orgânicos com 
diferentes graus de suscetibilidade à decomposição, oriundos da vegetação nativa com maior diversidade de espécies (PORTUGAL et al., 2008; CARDOSO et al., 2009). Adicionalmente, o estudo de Lima et al. (2011) em dois SAFs com seis e dez anos de implantação demonstrou que os sistemas aportaram mais $\mathrm{C}$ e $\mathrm{N}$ no solo em relação à vegetação nativa, e os autores atribuíram tal resultado à eficiência do manejo agroflorestal adotado, condicionando melhoria na qualidade do solo.

Assim, quanto maior o tempo de adoção de um SAF maior será a conservação da qualidade química do solo e maior o equilíbrio desse sistema, baseado na diversidade dos seus componentes e no arranjo estrutural que o aproxima de uma vegetação nativa (IWATA et al., 2012).

\section{Conclusão}

O manejo conservacionista dos sistemas agroflorestais contribuiu para a manutenção da densidade do solo em níveis semelhantes a vegetação nativa, e ao longo do tempo promoveu melhoria da fertilidade do solo na camada superficial.

Houve manutenção dos teores e estoques de C do solo sob sistemas agroflorestais que apresentaram maior diversidade florística e foram implantados com preservação das espécies arbóreas. Por outro lado, o sistema implantado com supressão da vegetação apresentou redução da qualidade do solo.

Houve incrementos nos teores e estoques de $\mathrm{N}$ no sistema agroflorestal com cultivo anual do feijão (Phaseolus vulgaris), espécie leguminosa que favorece a associação rizosférica com bactérias fixadoras de $\mathrm{N}_{2}$.

\section{Agradecimentos}

À Fundação de Amparo à Pesquisa do Estado de Minas Gerais (Fapemig), Conselho Nacional de Desenvolvimento Científico e Tecnológico (CNPq), e ao Programa de Cooperação Acadêmica (PROCAD/CAPES - 88881.068513/2014-01), pelo suporte financeiro. À Coordenação de Aperfeiçoamento de Pessoal de Nível Superior (CAPES) pela concessão de bolsa de mestrado à J. M. Ribeiro.

\section{Referências}

ALVARENGA, A. C. et al. Producción, composición y aporte de nutrientes de hojarasca en sistemas agroforestales. Spanish Journal of Rural Development, Lugo, v. 6, p. 85-100, 2015.

ALVARES, C. A. et al. Köppen's climate classification map for Brazil. Meteorologische Zeitschrift, Stuttgart, v. 22, p. 711-728, 2013.

ALVAREZ, V. V. H. et al. Interpretação dos resultados das análises de solos. In: RIBEIRO, A. C. et al. (org.). Recomendações para o uso de corretivos e fertilizantes em Minas Gerais: 5á Aproximação. 5. ed. Viçosa, MG: Comissão de Fertilidade do Solo do Estado de Minas Gerais, 1999. $360 \mathrm{p}$.

ALVES, B. J. R. et al. Determinação do nitrogênio em solo e planta. In: HUNGRIA, M.; ARAUJO, R. S. Manual de Métodos Empregados em Estudos de Microbiologia Agrícola. Brasília: EMBRAPA Arroz e Feijão, 1994. 542 p.

BOCHNER, J. K. et al. Matéria orgânica e agregação de um planossolo sob diferentes coberturas florestais. Cerne, Lavras, v. 14, n. 1, p. 46-53, 2008.

CAMPANHA, M. M.; SANTOS, R. H. S. Análise comparativa das características da serrapilheira e do solo em cafezais (Coffea arabica L.) cultivados em sistema agroflorestal e em monocultura na 
zona da mata-MG. Revista Árvore, Viçosa, MG, v. 31, n. 5, p. 805-812, 2007.

CARDOSO, E. L. et al. Atributos biológicos indicadores da qualidade do solo em pastagem cultivada e nativa no Pantanal. Pesquisa Agropecuária Brasileira, Brasília, v. 44, n. 6, p. 631637, 2009.

EMBRAPA. Centro Nacional de Pesquisas de Solos. Manual de Métodos de Análise de Solo. Rio de Janeiro: Ministério da Agricultura, 1997. 212 p.

FROUFE, L. C. M.; RACHWAL, M. F. G.; SEOANE, C. E. S. Potencial de sistemas agroflorestais multiestrata para sequestro de carbono de áreas de ocorrência de Floresta Atlântica. Pesquisa Florestal Brasileira, Colombo, v. 31, n. 66, p. 143-154, 2011.

GAMA-RODRIGUES, E. F. et al. Carbon storage in soil size fractions under two cacao agroforestry systems in Bahia, Brazil. Environmental Management, New York, n. 2, v. 45, p. 274-283, 2010.

IWATA, B. F. Dinâmica da matéria orgânica em argissolo vermelho amarelo sob sistemas agroflorestais no cerrado piauiense. 2010. Dissertação (Mestrado) - Universidade Federal do Piauí, Teresinha, 2010.

IWATA, B. F. et al. Sistemas agroflorestais e seus efeitos sobre os atributos químicos em Argissolo Vermelho-Amarelo do Cerrado piauiense. Revista Brasileira de Engenharia Agrícola Ambiental, Campina Grande, v. 16, n. 7, p. 730-738, 2012.

LEAL FILHO, N. et al. Comparando técnicas de nucleação utilizadas na restauração de áreas degradadas na Amazônia brasileira. Revista Árvore, Viçosa, MG, v. 37, n. 4, p. 587-597, 2013.

LIMA, S. S. et al. Atributos químicos e estoques de carbono e nitrogênio em argissolo vermelhoamarelo sob sistemas agroflorestais e agricultura de corte e queima no norte do Piauí. Revista Árvore, Viçosa, MG, v. 35, p. 51-60, 2011.

MAIA, S. M. F. et al. Impactos de sistemas agroflorestais e convencional sobre a qualidade do solo no semi-árido cearense. Revista Árvore, Viçosa, MG, v. 30, n. 5, p. 837-848, 2006.

MATIAS, S. S. R. et al. Atributos físicos de um Latossolo Vermelho submetido a diferentes usos. Revista Ciência Agronômica, Fortaleza, v. 40, n. 3, p. 331-338, 2009.

PITOMBO, L. M. Estoques de carbono e nitrogênio e fluxos de gases do efeito estufa em solo com diferentes históricos de aplicação de lodo de esgoto. 2011. Dissertação (Mestrado) Instituto Agronômico de Campinas, Campinas, 2011.

PORTUGAL, A. F. et al. Atributos químicos e físicos de um Cambissolo Háplico Tb distrófico sob diferentes usos na Zona da Mata mineira. Revista Brasileira de Ciência do Solo, Viçosa, MG, v. 32, p. 249-258, 2008.

PULROLNIK, K. et al. Estoques de carbono e nitrogênio em frações lábeis e estáveis da matéria orgânica de solos sob eucalipto, pastagem e cerrado no vale do Jequitinhonha-MG. Revista Brasileira de Ciência do Solo, Viçosa, MG, v. 33, p. 1125-1136, 2009.

ROCHA, G. P. et al. Caracterização e estoques de carbono de sistemas agroflorestais no Cerrado de Minas Gerais. Ciência Rural, Santa Maria, v. 44, n. 7, p. 1197-1203, 2014.

SANTIAGO, W. R. et al. Nitrogênio mineral e microbiano do solo em sistemas agroflorestais com palma de óleo na Amazônia oriental. Acta Amazônica, Manaus, v. 43, n. 4, p. 395-406, 2013.

SILVA, A. T. Sistema agroflorestal sobre cultivo de leguminosas: fertilidade do solo, resistência a penetração e produtividade de milho e feijão-caupi. 2011. Dissertação (Mestrado) - Universidade Federal do Tocantins, Gurupi, 2011.

SILVA, C. A.; VALE, F. R. Disponibilidade de nitrato em solos brasileiros sob efeito da calagem e de fontes e doses de nitrogênio. Pesquisa Agropecuária Brasileira, Brasília, v. 35, n. 12, p. 2461- 
$2471,2000$.

SILVA, C. F. et al. Carbono orgânico total, biomassa microbiana e atividade enzimática do solo de áreas agrícolas, florestais e pastagem no médio vale do Paranaíba do Sul (RJ). Revista Brasileira de Ciência do Solo, Viçosa, MG, v. 36, p. 1680-1689, 2012.

SILVA, I. F.; ARAÚJO, S. E.; KUSDRA, J. F. Biological activity of soils under systems of organic farming, agroforestry and pasture in the Amazon. Revista Ciência Agronômica, Fortaleza, v. 45, n. 3, p. 427-432, 2014. 